\title{
Selective survival advantage associated with primary tumor resection for metastatic gastric cancer in a Western population
}

\author{
René Warschkow $^{1} \cdot$ Matthias Baechtold $^{2} \cdot$ Kenneth Leung $^{3} \cdot$ Bruno M. Schmied $^{1}$ • \\ Daniel P. Nussbaum ${ }^{3} \cdot$ Beat Gloor $^{2} \cdot$ Dan G. Blazer $\mathrm{III}^{3} \cdot$ Mathias Worni $^{2}(\mathbb{D}$
}

Received: 24 December 2016/Accepted: 12 June 2017/Published online: 23 June 2017

(c) The International Gastric Cancer Association and The Japanese Gastric Cancer Association 2017

\begin{abstract}
Background The prognosis of metastatic gastric cancer (GC) remains dismal, with a median survival of 10 months. Historically, primary tumor resection was not thought to confer any survival benefit. Although high-level data exist guiding treatment of metastatic GC for patients in the East, no such data exist for Western patients despite inherent ethnic differences in GC biology.

Methods The 2006-2012 National Cancer Database was queried for adult patients with metastatic gastric adenocarcinoma. Patients were classified into those who underwent primary tumor resection and chemotherapy (PTRaC) and those who received chemotherapy only. Groups were propensity score matched, and survival was compared using advanced statistical modeling.

Results A total of 7026 patients met the inclusion criteria: 6129 (87\%) patients were treated with chemotherapy alone and $897(13 \%)$ patients were treated with PTRaC. After multivariable adjustment, patients who underwent PTRaC had a significantly better overall survival (OS) than patients who received systemic therapy only (HR, $0.60 ; 95 \%$ CI, $0.56-0.64 ; p<0.001)$. Following full bipartite propensity
\end{abstract}

René Warschkow and Matthias Baechtold contributed equally to this paper.

Mathias Worni

mathias.worni@insel.ch

1 Department of Surgery, Kantonsspital St. Gallen, 9007 St. Gallen, Switzerland

2 Department of Visceral and Transplantation Surgery, Inselspital, Bern University Hospital, University of Bern, 3010 Bern, Switzerland

3 Department of Surgery, Duke University Medical Center, Duke University, Durham, NC, USA score-adjusted analysis, 2-year OS for patients who received chemotherapy only was $12.6 \% \quad(95 \% \quad \mathrm{CI}$, 11.7-13.5\%), whereas it was 34.2\% (95\% CI, 31.3-37.5\%) for patients who underwent PTRaC (HR for resection: 0.52; 95\% CI, 0.47-0.57; $p<0.001)$.

Conclusion Our data suggest that there exists a subset of patients with metastatic GC for which PTRaC may improve OS. As significant uncertainty still remains, our results support the need for further prospective trials investigating the influence of palliative gastrectomy on survival among Western patients.

Keywords Gastric cancer · Palliative gastrectomy · Overall survival · Primary tumor resection · Metastatic

\section{Introduction}

Globally, gastric cancer (GC) is the fifth most common cancer worldwide, contributing significantly to the burden of cancer-related morbidity and mortality [1-3]. Although less prevalent in Western countries, an estimated 26,500 new cases of GC are expected in the United States in 2016 [4]. In contrast to Asian countries, many of which have screening programs in place $(53 \%$ of the GC patients are diagnosed with localized disease), in Western countries GC is often diagnosed at a more advanced stage [5]. In the US, approximately two-thirds of patients are diagnosed with stage III or IV disease, and of these, 34\% will have metastatic disease [6, 7]. When GC is detected at a localized stage, it is widely accepted that resection combined with perioperative chemotherapy or chemoradiotherapy offers the best chance for long-term survival [8, 9]. However, the optimal treatment strategy for metastatic GC 
remains a topic of debate, in part because the impact of primary tumor resection on survival is not clear [7, 10].

The prognosis of metastatic GC is dismal, with a 5-year overall survival (OS) of $4 \%$ and a median survival of 10 months with chemotherapy alone [11]. Historically, these patients underwent palliative systemic therapy as the perioperative risk was significant and the benefit of primary tumor resection was thought to be minimal. Through advances in patient selection, perioperative care, and surgical technique, perioperative mortality has fallen to approximately $1 \%$; this improved rate has renewed interest in evaluating the effects of primary tumor resection on overall survival in patients with metastatic GC [12-14]. The Dutch Gastric Cancer Trial was the first study suggesting that gastrectomy for patients with metastatic GC has the potential to improve survival compared to patients undergoing nonresectional surgery [14]. A populationbased analysis using the Surveillance Epidemiology and End Results (SEER) cancer registry showed a modest OS improvement in the context of a primary tumor resection for stage IV patients [15]. In contrast, one multicenter randomized controlled trial conducted in Asia, the REGATTA trial, did not show any benefit of gastrectomy on OS [13].

However, because of distinct differences in GC between Eastern and Western populations, it is questionable whether the results from the REGATTA trial can be directly applied to Western countries [16-21]. For instance, it is known that the age-adjusted incidence rate between Eastern and Western countries differs as much as fourfold; furthermore, Asian patients often have more distal tumors and lower-grade tumors, and Asian patients undergo more extensive lymphadenectomy compared to their counterparts. Even after adjusting for those well-known prognostic factors, Asian race remains a strong independent predictor of improved survival in a population-based study of Los Angeles County in the US [19]. Further research is needed to better delineate reasons for those ethnic differences; however, recognition that these differences exist suggest that Eastern studies may not be immediately generalizable to the West. Given the paucity of randomized data, we sought to utilize a large nationwide clinical oncology database to evaluate therapy related to primary tumor resection in stage IV GC in Western patients.

The primary aim of this study was to assess whether primary tumor resection and chemotherapy (PTRaC) had the potential to impact OS among patients with stage IV GC. Secondary aims were to characterize patients undergoing PTRaC as well as those who are likely to benefit from surgical resection of the primary tumor.

\section{Methods}

\section{Data source}

The National Cancer Database (NCDB) is jointly maintained by the American College of Surgeons Commission on Cancer (ACS CoC) and the American Cancer Society and gathers data from more than 1500 academic and community centers. The NCDB is estimated to capture $70 \%$ of all newly diagnosed cases of cancer in the United States and Puerto Rico and now contains more than 30 million patient records [22].

Patients aged 18 years and older diagnosed from 2006 to 2012 with histologically confirmed metastatic (stage IV) adenocarcinoma of the stomach, as defined by the 7th edition of the American Joint Commission on Cancer (AJCC), were included in this study. Patients with adenocarcinomas of the stomach were identified using the following International Classification of Diseases in Oncology (ICD-O-3) histology codes: 8140-8145, 8210-8211, 8255-8323, and 8470-8490 [23]. Given the inherent difference of the treatment strategy for patients with gastric cardia carcinoma, these patients were excluded from the analyses. In addition, patients with additional primary cancer diagnoses other than GC, patients undergoing surgery outside the primary tumor site (e.g., resection of distant metastatic disease), or unknown surgical information and with unknown information about radiation therapy were excluded to form a homogeneous cohort for further analysis. Finally, patients who did not undergo surgical resection of the primary tumor and did not receive chemotherapy were also excluded. To assess the effect of surgical resection of the primary tumor among metastatic GC patients, our investigated patients were divided into two groups: (1) undergoing surgical resection of the primary tumor with chemotherapy and (2) receiving chemotherapy only. To account for metastatic tumor burden based on available information within the NCDB, we grouped the metastatic sites as follows: (1) distant lymph nodes, (2) peritoneum only, (3) peritoneum \pm solid organ, (4) distant lymph nodes and peritoneum \pm solid organ, and (5) unknown metastatic site. Based on inherent changes in coding within the NCDB over time, it was not possible to further delineate whether solid organ metastases were present for some patients with known peritoneal metastases (group 3). The following outcome variables were extracted from the NCDB: length of hospital stay, 30-day postoperative re-hospitalization rate, 30-day and 90-day postoperative mortality, and overall survival (in relation to date of diagnosis). 


\section{Statistical analysis}

Statistical analyses were performed using $\mathrm{R}$ statistical software (http://www.r-project.org). A two-sided $p$ value $<0.05$ was considered statistically significant. Continuous data were expressed as mean \pm standard deviation and categorical variables as counts (percentage). Survival data were presented as overall, 1-year, 2-year, or median survival, as appropriate. Weighted and unweighted chi-square statistics were used to analyze proportions. Time trends were assessed by Spearman rank correlation analysis. In regression analysis, $p$ values were computed by likelihood ratio tests, and Wald-type confidence intervals were estimated.

After comparing baseline treatment and outcome data, predictors of primary tumor resection were assessed by univariable and multivariable logistic regression with adjustment for additional variables: tumor location [fundus, corpus, antrum/pylorus, curvatures, other (overlapping and unspecified lesions)], tumor grade (G1/2, G3/4, unknown), histological subtype (adenocarcinoma/other, intestinal, diffuse type/linitis plastica, signet-cell), type of chemotherapy (single-agent, multiple-agent, unknown), radiation therapy (yes, no), year of diagnosis (2006-2008, 2009-2010, 2011-2012), age $(<50,50-64,65-79$, $80+$ years), gender (male, female), race (white, black, other/unknown), Charlson-Deyo comorbidity score $(0,1$, $\geq 2$ ), type of surgery (none, partial gastrectomy, total gastrectomy, gastrectomy with esophagus, gastrectomy with other organ, gastrectomy not otherwise specified), resection margin defined by the definitive pathological margin after resection of the primary tumor (R0, negative; $\mathrm{R}+$, microscopically or macroscopically positive; Rx, unknown; no surgery to the primary tumor), chemotherapy before primary tumor resection (yes, no, other), distance to facility in miles (mean), and urban living (yes, no). Thereafter, survival was assessed by univariable Cox regression. Proportionality assumption was tested by scaling Schoenfeld residuals and inspecting the hazard ratio plots [24]. To account for the inherent selection bias, three strategies were applied. First, a multivariable Cox regression with adjustment for additional variables was performed. Second, full bipartite weighted and matched propensity score analysis with stratification for the year of diagnosis was performed using the "MatchIt" and the "optmatch" packages $[25,26]$. Within the matched cohort, the baseline characteristics were compared to ensure that no major difference persisted. Third, an exact matching and weighting analysis was performed such that identical distributions of the baseline characteristics resulted [25]. The weights and subclasses obtained by the two propensity score procedures were included in Cox regression analyses. Patients without a counterpart with similar baseline characteristics in the comparison group were excluded from these analyses.

To assess predictors of long-term survival (defined as a survival of 2 years) among patients who underwent primary tumor resection, multivariable adjusted logistic regression analyses were performed. Subgroup analyses were performed after left-hand truncation of 3 months to exclude patients with very poor prognosis. In addition, subgroup analyses were performed after exclusion of patients younger than 20 years or older than 75 years. Furthermore, subgroup analyses were performed for patients with distant lymph node metastases only and for peritoneal metastases only. Based on information within NCDB, no subgroup analysis was possible for patients with liver metastases only.

\section{Results}

\section{Patient cohort and short-term outcomes after surgery}

A total of 7026 patients met the study inclusion criteria (Fig. 1). Of those, 6129 patients were treated with chemotherapy alone and 897 were treated with PTRaC. The two groups were heterogeneous in several patient and tumor characteristics; however, no differences were found in regard to comorbidities, urban living, or distance to treatment facility (Table 1). Most patients who underwent a primary tumor resection had a partial gastrectomy (46.5\%), followed by gastrectomy with other organs $(20.5 \%)$ or total gastrectomy (19.7\%). The rate of patients who underwent primary tumor resection decreased with increasing tumor burden. Most patients with a primary tumor resection received chemotherapy after surgery $(75.5 \%)$ but only a few patients had systemic therapy both before and after surgery $(6.9 \%)$. The rate of patients who underwent primary tumor resection decreased over time from $17 \%$ to $11 \%$ during the study period ( $p$ for trend: 0.03), whereas the use of multiagent chemotherapy increased from $78 \%$ to $85 \%$ ( $p$ for trend: $<0.001$ ). Mean length of hospital stay after surgery was 10.2 days (SD, 8.5), with an unplanned 30-day re-hospitalization rate of $5.7 \%$. Thirty- and 90-day postoperative mortality rates were $1.3 \%$ and $8.2 \%$, respectively. Mean time from diagnosis to primary tumor resection was 51.7 days (SD, 78.9), and $54.3 \%$ of the patients had a margin-negative resection (R0).

\section{Predictors of primary tumor resection}

After multivariable adjustment, patients who underwent PTRaC were more likely to be female, between ages of 50 and 64 years, with a tumor within the antrum/pyloric 


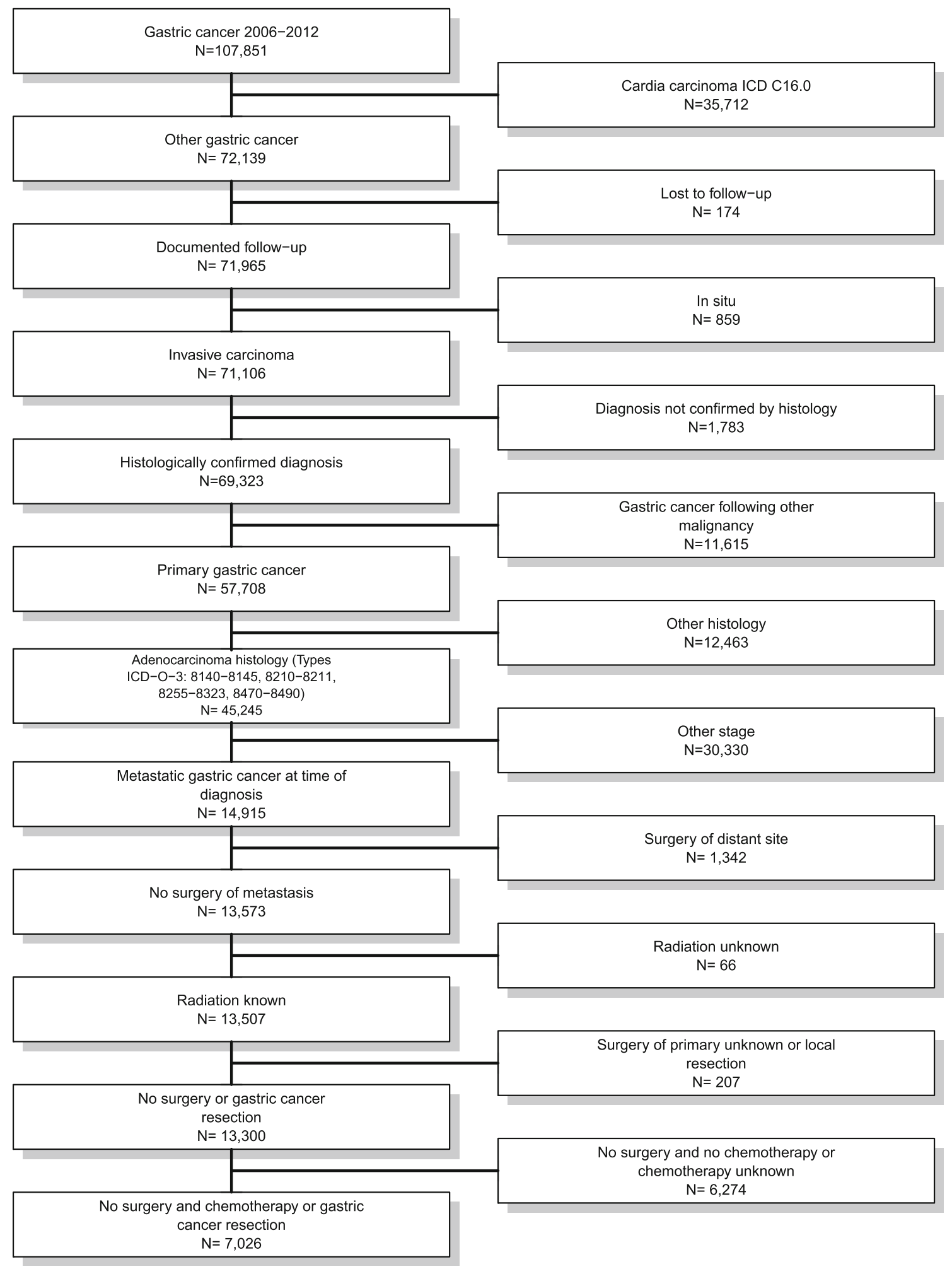

Fig. 1 Flowchart of patient selection

region or within the curvatures compared to the fundus, and to have received radiation therapy. Patients with peritoneal with/without solid organ or distant lymph nodes and peritoneum with/without solid organ metastases were less likely to undergo surgery compared to patients with isolated peritoneal metastases or distant lymph node metastases only, those with typical adenocarcinoma histology, or who had received multi-agent chemotherapy (Table 2). 
Table 1 Baseline patient characteristics: treatment and short-term outcome data

\begin{tabular}{|c|c|c|c|c|}
\hline & $\begin{array}{l}\text { Total } \\
n=7026\end{array}$ & $\begin{array}{l}\text { Chemotherapy } \\
\text { only } \\
n=6129\end{array}$ & $\begin{array}{l}\text { Chemotherapy and gastrectomy } \\
n=897\end{array}$ & $p$ value \\
\hline \multicolumn{5}{|l|}{ Gender } \\
\hline Male & $4203(59.8)$ & $3698(60.3)$ & $505(56.3)$ & \multirow[t]{2}{*}{$0.021^{\mathrm{b}}$} \\
\hline Female & $2823(40.2)$ & $2431(39.7)$ & $392(43.7)$ & \\
\hline \multicolumn{5}{|l|}{ Age group } \\
\hline$<50$ years & $1549(22.0)$ & $1366(22.3)$ & $183(20.4)$ & \multirow[t]{4}{*}{$0.009^{\mathrm{b}}$} \\
\hline 50-64 years & $2660(37.9)$ & $2280(37.2)$ & $380(42.4)$ & \\
\hline $65-79$ years & $2327(33.1)$ & $2040(33.3)$ & $287(32.0)$ & \\
\hline $80+$ years & $490(7.0)$ & $443(7.2)$ & $47(5.2)$ & \\
\hline \multicolumn{5}{|l|}{ Charlson-Deyo Score } \\
\hline 0 & $5313(75.6)$ & $4637(75.7)$ & $676(75.4)$ & \multirow[t]{3}{*}{$0.98^{\mathrm{b}}$} \\
\hline 1 & $1321(18.8)$ & $1150(18.8)$ & $171(19.1)$ & \\
\hline$\geq 2$ & $392(5.6)$ & $342(5.6)$ & $50(5.6)$ & \\
\hline \multicolumn{5}{|l|}{ Race } \\
\hline White & $4827(68.7)$ & $4259(69.5)$ & $568(63.3)$ & \multirow[t]{3}{*}{$<0.001^{\mathrm{b}}$} \\
\hline Black & $1421(20.2)$ & $1220(19.9)$ & $201(22.4)$ & \\
\hline Other/unknown & $778(11.1)$ & $650(10.6)$ & $128(14.3)$ & \\
\hline \multicolumn{5}{|l|}{ Year of diagnosis (three groups) } \\
\hline 2006-2008 & $2723(38.8)$ & $2293(37.4)$ & $430(47.9)$ & \multirow[t]{3}{*}{$<0.001^{\mathrm{b}}$} \\
\hline 2009-2010 & $2020(28.8)$ & $1794(29.3)$ & $226(25.2)$ & \\
\hline 2011-2012 & $2283(32.5)$ & $2042(33.3)$ & $241(26.9)$ & \\
\hline \multicolumn{5}{|l|}{ Chemotherapy type } \\
\hline Single agent & $797(11.3)$ & $675(11.0)$ & $122(13.6)$ & \multirow[t]{3}{*}{$<0.001^{\mathrm{b}}$} \\
\hline Multiple agents & $5743(81.7)$ & $5057(82.5)$ & $686(76.5)$ & \\
\hline Agent unknown & $486(6.9)$ & $397(6.5)$ & $89(9.9)$ & \\
\hline \multicolumn{5}{|l|}{ Radiation therapy } \\
\hline No & $6075(86.5)$ & $5336(87.1)$ & $739(82.4)$ & \multirow[t]{2}{*}{$<0.001^{\mathrm{b}}$} \\
\hline Yes & $951(13.5)$ & $793(12.9)$ & $158(17.6)$ & \\
\hline \multicolumn{5}{|l|}{ Metastasis } \\
\hline Distant LNs only & $679(9.7)$ & $554(9.0)$ & $125(13.9)$ & \multirow[t]{5}{*}{$<0.001^{\mathrm{b}}$} \\
\hline Peritoneal only & $959(13.6)$ & $817(13.3)$ & $142(15.8)$ & \\
\hline Peritoneal \pm solid organ & $3583(51.0)$ & $3113(50.8)$ & $470(52.4)$ & \\
\hline Distant LNs and peritoneal \pm solid organ & $1169(16.6)$ & $1101(18.0)$ & $68(7.6)$ & \\
\hline Unknown & $636(9.1)$ & $544(8.9)$ & $92(10.3)$ & \\
\hline \multicolumn{5}{|l|}{ Histology } \\
\hline Adenocarcinoma NOS & $4419(62.9)$ & $3956(64.5)$ & $463(51.6)$ & \multirow[t]{4}{*}{$<0.001^{\mathrm{b}}$} \\
\hline Intestinal & $353(5.0)$ & $265(4.3)$ & $88(9.8)$ & \\
\hline Diffuse type, linitis plastica & $327(4.7)$ & $252(4.1)$ & $75(8.4)$ & \\
\hline Signet-ring cell features & $1927(27.4)$ & $1656(27.0)$ & $271(30.2)$ & \\
\hline \multicolumn{5}{|l|}{ Tumor grade } \\
\hline Grade $1 / 2$ & 1154 (16.4) & $1002(16.3)$ & $152(16.9)$ & \multirow[t]{3}{*}{$<0.001^{\mathrm{b}}$} \\
\hline Grade $3 / 4$ & $4403(62.7)$ & $3698(60.3)$ & $705(78.6)$ & \\
\hline Unknown & $1469(20.9)$ & $1429(23.3)$ & $40(4.5)$ & \\
\hline \multicolumn{5}{|l|}{ Tumor location } \\
\hline Fundus & $496(7.1)$ & $462(7.5)$ & $34(3.8)$ & \multirow[t]{3}{*}{$<0.001^{\mathrm{b}}$} \\
\hline Corpus & $920(13.1)$ & $826(13.5)$ & $94(10.5)$ & \\
\hline Antrum/pylorus & $1456(20.7)$ & $1173(19.1)$ & $283(31.5)$ & \\
\hline
\end{tabular}


Table 1 continued

\begin{tabular}{|c|c|c|c|c|}
\hline & $\begin{array}{l}\text { Total } \\
n=7026\end{array}$ & $\begin{array}{l}\text { Chemotherapy } \\
\text { only } \\
n=6129\end{array}$ & $\begin{array}{l}\text { Chemotherapy and gastrectomy } \\
n=897\end{array}$ & $p$ value \\
\hline Lesser/greater curvature & $940(13.4)$ & $793(12.9)$ & $147(16.4)$ & \\
\hline Other & $3214(45.7)$ & $2875(46.9)$ & $339(37.8)$ & \\
\hline \multicolumn{5}{|l|}{ Timing of chemotherapy } \\
\hline No chemotherapy before primary tumor resection & NA & NA & $677(75.5)$ & \\
\hline Chemotherapy before primary tumor resection & & & $220(24.5)$ & \\
\hline \multicolumn{5}{|l|}{ Performed surgery } \\
\hline No surgery & $6129(87.2)$ & $6129(100.0)$ & $0(0.0)$ & \\
\hline Partial gastrectomy & $417(5.9)$ & $0(0.0)$ & $417(46.5)$ & \\
\hline Total gastrectomy & $177(2.5)$ & $0(0.0)$ & $177(19.7)$ & \\
\hline Gastrectomy and esophagus & $102(1.5)$ & $0(0.0)$ & $102(11.4)$ & \\
\hline Gastrectomy and other organs & $184(2.6)$ & $0(0.0)$ & $184(20.5)$ & \\
\hline Gastrectomy NOS & $17(0.2)$ & $0(0.0)$ & $17(1.9)$ & \\
\hline \multicolumn{5}{|l|}{ Resection margin } \\
\hline $\mathrm{R} 0$ & $479(6.8)$ & $0(0.0)$ & $479(53.4)$ & $<0.001^{\mathrm{b}}$ \\
\hline $\mathrm{R}+$ & $381(5.4)$ & $0(0.0)$ & $381(42.5)$ & \\
\hline $\mathrm{Rx}$ & $37(0.5)$ & $0(0.0)$ & $37(4.1)$ & \\
\hline No surgery & $6129(87.2)$ & $6129(100.0)$ & $0(0.0)$ & \\
\hline \multicolumn{5}{|l|}{ Number of lymph nodes resected } \\
\hline $0-11$ & NA & NA & $352(39.2)$ & \\
\hline $12+$ & & & $519(57.9)$ & \\
\hline \multicolumn{5}{|l|}{ Urban $^{\mathrm{a}}$} \\
\hline Metropolitan & $5884(83.7)$ & $5138(83.8)$ & $746(83.2)$ & $0.61^{\mathrm{b}}$ \\
\hline Non-metropolitan & $1142(16.3)$ & $991(16.2)$ & $151(16.8)$ & \\
\hline Distance to treatment facility in miles (mean, SD) & $\begin{array}{l}27.1 \\
(129.6)\end{array}$ & $26.8(129.6)$ & $29.4(130.0)$ & $0.35^{\mathrm{c}}$ \\
\hline Length of hospital stay postoperative days (mean, SD) & NA & NA & $10.2(8.5)$ & \\
\hline Unknown & & & $112(12.5)$ & \\
\hline \multicolumn{5}{|l|}{ 30-day mortality postoperative } \\
\hline No & NA & NA & $882(98.3 \%)$ & \\
\hline Yes & & & $12(1.3 \%)$ & \\
\hline Unknown & & & $3(0.3 \%)$ & \\
\hline \multicolumn{5}{|l|}{ 90-day mortality postoperative } \\
\hline No & NA & NA & $812(90.5)$ & \\
\hline Yes & & & $74(8.2)$ & \\
\hline Unknown & & & $11(1.2)$ & \\
\hline $\begin{array}{l}\text { Time from diagnosis to treatment start days (mean, } \\
\text { SD) }\end{array}$ & $31.1(42.3)$ & $32.5(42.7)$ & $21.6(37.7)$ & $<0.001^{\mathrm{c}}$ \\
\hline Unknown & $380(5.4)$ & $326(5.3)$ & $54(6.0)$ & \\
\hline Length of follow-up years (mean, SD) & $1.0(1.0)$ & $0.9(0.9)$ & $1.5(1.3)$ & $<0.001^{\mathrm{c}}$ \\
\hline
\end{tabular}

Data are shown as number of patients $n(\%)$ or as mean (SD), as indicated

${ }^{a}$ Cities with more than 0.25 million inhabitants were defined as metropolitan

b Chi-square test

c Mann-Whitney $U$ test

NOS not otherwise specified, $N A$ not applicable, $L N$ lymph node 
Table 2 Predictors of primary tumor resection among metastatic gastric cancer patients

\begin{tabular}{|c|c|c|c|c|}
\hline & \multicolumn{2}{|c|}{ Unadjusted logistic regression $^{\mathrm{a}}$} & \multicolumn{2}{|c|}{ Multivariable adjusted logistic regression ${ }^{\mathrm{b}}$} \\
\hline & OR $(95 \% \mathrm{CI})$ & $p$ value* & OR $(95 \% \mathrm{CI})$ & $p$ value* \\
\hline \multicolumn{5}{|l|}{ Gender } \\
\hline Male & Reference & 0.022 & Reference & 0.032 \\
\hline Female & $1.18(1.02-1.36)$ & & $1.18(1.01-1.37)$ & \\
\hline \multicolumn{5}{|l|}{ Age group } \\
\hline$<50$ years & Reference & 0.008 & Reference & $<0.001$ \\
\hline $50-64$ years & $1.24(1.03-1.50)$ & & $1.37(1.12-1.67)$ & \\
\hline $65-79$ years & $1.05(0.86-1.28)$ & & $1.14(0.92-1.41)$ & \\
\hline $80+$ years & $0.79(0.56-1.10)$ & & $0.72(0.49-1.03)$ & \\
\hline \multicolumn{5}{|l|}{ Race } \\
\hline White & Reference & $<0.001$ & Reference & 0.049 \\
\hline Black & $1.24(1.04-1.47)$ & & $1.14(0.95-1.37)$ & \\
\hline Other/unknown & $1.48(1.19-1.81)$ & & $1.30(1.04-1.62)$ & \\
\hline \multicolumn{5}{|l|}{ Year of diagnosis } \\
\hline $2006-2008$ & Reference & $<0.001$ & Reference & $<0.001$ \\
\hline $2009-2010$ & $0.67(0.56-0.80)$ & & $0.57(0.47-0.69)$ & \\
\hline 2011-2012 & $0.63(0.53-0.74)$ & & $0.45(0.36-0.56)$ & \\
\hline \multicolumn{5}{|l|}{ Charlson-Deyo Score } \\
\hline 0 & Reference & 0.98 & Reference & 0.87 \\
\hline 1 & $1.02(0.85-1.22)$ & & $1.03(0.85-1.24)$ & \\
\hline$\geq 2$ & $1.00(0.73-1.35)$ & & $1.09(0.78-1.50)$ & \\
\hline \multicolumn{5}{|l|}{ Tumor location } \\
\hline Fundus & Reference & $<0.001$ & Reference & $<0.001$ \\
\hline Corpus & $1.55(1.04-2.36)$ & & $1.33(0.88-2.05)$ & \\
\hline Antrum/pylorus & $3.28(2.29-4.83)$ & & $3.11(2.15-4.63)$ & \\
\hline Curvatures & $2.52(1.73-3.78)$ & & $2.34(1.58-3.54)$ & \\
\hline Other & $1.60(1.13-2.35)$ & & $1.45(1.01-2.15)$ & \\
\hline \multicolumn{5}{|l|}{ Tumor grade } \\
\hline Grade $1 / 2$ & Reference & $<0.001$ & Reference & $<0.001$ \\
\hline Grade $3 / 4$ & $1.26(1.04-1.52)$ & & $1.22(0.99-1.51)$ & \\
\hline Unknown & $0.18(0.13-0.26)$ & & $0.18(0.13-0.26)$ & \\
\hline \multicolumn{5}{|l|}{ Radiation therapy } \\
\hline No & Reference & $<0.001$ & Reference & $<0.001$ \\
\hline Yes & $1.44(1.19-1.73)$ & & $1.42(1.15-1.73)$ & \\
\hline \multicolumn{5}{|l|}{ Metastasis } \\
\hline Distant LNs only & Reference & $<0.001$ & Reference & $<0.001$ \\
\hline Peritoneal only & $0.77(0.59-1.00)$ & & $1.22(0.91-1.64)$ & \\
\hline Peritoneal \pm solid organ & $0.67(0.54-0.83)$ & & $0.67(0.53-0.84)$ & \\
\hline Distant LNs and peritoneum \pm solid organ & $0.27(0.20-0.37)$ & & $0.30(0.22-0.42)$ & \\
\hline Unknown & $0.75(0.56-1.00)$ & & $1.18(0.86-1.63)$ & \\
\hline \multicolumn{5}{|l|}{ Histology } \\
\hline Adenocarcinoma NOS & Reference & $<0.001$ & Reference & $<0.001$ \\
\hline Intestinal & $2.84(2.18-3.66)$ & & $2.84(2.13-3.75)$ & \\
\hline Diffuse type, linitis plastica & $2.54(1.92-3.33)$ & & $2.49(1.84-3.33)$ & \\
\hline Signet-ring cell features & $1.40(1.19-1.64)$ & & $1.40(1.18-1.67)$ & \\
\hline \multicolumn{5}{|l|}{ Chemotherapy type } \\
\hline Single agent & Reference & $<0.001$ & Reference & $<0.001$ \\
\hline Multiple agents & $0.75(0.61-0.93)$ & & $0.78(0.62-0.98)$ & \\
\hline Agent unknown & $1.24(0.92-1.67)$ & & $1.32(0.95-1.82)$ & \\
\hline
\end{tabular}

Odds ratios (OR) with 95\% confidence intervals (Wald type)

* $p$ from likelihood ratio test

a Univariate logistic regression analysis

b Multivariable adjusted logistic regression analysis

$N O S$ not otherwise specified, $L N$ lymph node 


\section{Survival analysis}

The median survival for the entire cohort was 8.5 months [95\% confidence interval (CI), 8.2-8.7 months], and the 2-year OS rate was $14.7 \%$ (95\% CI, 13.9-15.6\%). For patients who underwent systemic therapy, the median survival and the 2-year OS rate were 7.9 months $(95 \% \mathrm{CI}$, 7.7-8.1 months) and $13.1 \%$ (95\% CI, $12.2-14.1 \%)$, respectively, compared to 13.9 months $(95 \% \mathrm{CI}$, 13.0-14.8 months) and 25.4\% (95\% CI, 22.6-28.5\%), respectively, for patients who underwent PTRaC (Fig. 2a).

After multivariable adjustment, patients who had PTRaC had significantly better OS than patients who underwent systemic therapy only [hazard ratio (HR), 0.60, 95\% CI, $0.56-0.64 ; p<0.001$ ] (Table 3). Additionally, better OS was observed in patients with grade $1 / 2$ tumors, patients who received multi-agent chemotherapy, and patients with lower comorbidity scores, whereas patients with increasing metastatic tumor burden had worse OS.

The full bipartite propensity score-adjusted analysis was based on a total of 6455 patients, with 5570 patients within the chemotherapy group (exclusion of 559 patients) and 885 patients within the PTRaC group (exclusion of 12 patients). The 2-year OS of patients who received chemotherapy only was $12.6 \%$ (95\% CI, 11.7-13.5\%) compared to $34.2 \%$ (95\% CI, 31.3-37.5\%) for patients who underwent PTRaC (HR for resection: $0.52 ; 95 \% \mathrm{CI}$, $0.47-0.57 ; p<0.001)$ (Fig. 2b).

The exact matching and weighting adjusted analysis was based on a total of 1186 patients, with 853 patients within the chemotherapy group (exclusion of 5276 patients) and 333 patients within the PTRaC group (exclusion of 564 patients). The 2-year OS was $12.3 \%$ (95\% CI, 10.3-14.7\%) for the chemotherapy-only group and $27.8 \%$ (95\% CI, 23.6-32.9\%) for patients who underwent PTRaC (HR for resection: $0.61 ; 95 \% \mathrm{CI}, 0.53-0.71 ; p<0.001)$.

\section{Predictors of long-term survival ( $>2$ years) in patients who underwent primary tumor resection}

Among the 897 patients who underwent PTRaC, 100 patients survived at least 2 years after diagnosis. After multivariable adjustment, the following factors were associated with improved long-term survival: older age, race other than Caucasian, use of radiation therapy, and chemotherapy before and after surgery. In contrast, signet-ring cell features, peritoneal with/without solid organ metastases and distant lymph nodes and peritoneum with/without solid organ metastases, primary tumor location in the corpus and antrum, and positive resection margin were associated with worse long-term survival (Table 4).
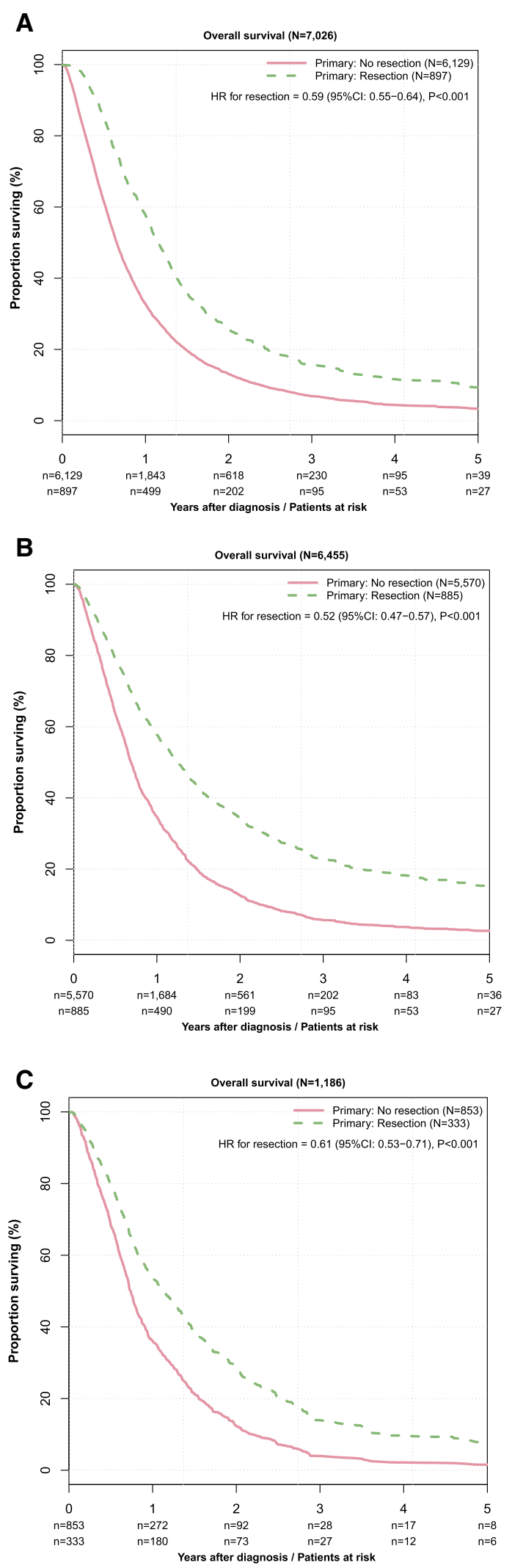

Fig. 2 Overall survival curves in unadjusted analysis (a), after full propensity score matching (b), and exact propensity score matching (c) 
Table 3 Univariate and multivariable adjusted overall survival analyses for patients with metastatic gastric cancer

\begin{tabular}{|c|c|c|c|c|}
\hline & \multicolumn{2}{|c|}{ Unadjusted Cox regression $^{\mathrm{a}}$} & \multicolumn{2}{|c|}{ Multivariable adjusted Cox regression ${ }^{\mathrm{b}}$} \\
\hline & HR $(95 \% \mathrm{CI})$ & $p$ value* & HR $(95 \%$ CI $)$ & $p$ value* \\
\hline \multicolumn{5}{|l|}{ Therapy } \\
\hline Chemotherapy only & Reference & \multirow[t]{2}{*}{$<0.001$} & Reference & \multirow[t]{2}{*}{$<0.001$} \\
\hline Resection and chemotherapy & $0.59(0.56-0.64)$ & & $0.60(0.56-0.64)$ & \\
\hline \multicolumn{5}{|l|}{ Gender } \\
\hline Male & Reference & \multirow[t]{2}{*}{0.40} & Reference & \multirow[t]{2}{*}{0.84} \\
\hline Female & $1.02(0.97-1.08)$ & & $1.01(0.95-1.06)$ & \\
\hline \multicolumn{5}{|l|}{ Age group } \\
\hline$<50$ years & Reference & \multirow[t]{4}{*}{$<0.001$} & Reference & \multirow[t]{4}{*}{$<0.001$} \\
\hline 50-64 years & $0.92(0.86-0.99)$ & & $0.95(0.88-1.01)$ & \\
\hline $65-79$ years & $0.97(0.91-1.04)$ & & $0.98(0.91-1.05)$ & \\
\hline $80+$ years & $1.24(1.11-1.39)$ & & $1.21(1.07-1.36)$ & \\
\hline \multicolumn{5}{|l|}{ Race } \\
\hline White & Reference & \multirow[t]{3}{*}{$<0.001$} & Reference & \multirow[t]{3}{*}{0.002} \\
\hline Black & $0.91(0.85-0.97)$ & & $0.95(0.89-1.02)$ & \\
\hline Other/unknown & $0.82(0.76-0.89)$ & & $0.87(0.80-0.94)$ & \\
\hline \multicolumn{5}{|l|}{ Year of diagnosis } \\
\hline $2006-2008$ & Reference & \multirow[t]{3}{*}{$<0.001$} & Reference & \multirow[t]{3}{*}{$<0.001$} \\
\hline 2009-2010 & $0.93(0.88-0.99)$ & & $0.91(0.85-0.97)$ & \\
\hline 2011-2012 & $0.83(0.78-0.88)$ & & $0.81(0.75-0.87)$ & \\
\hline \multicolumn{5}{|l|}{ Charlson-Deyo Score } \\
\hline 0 & Reference & \multirow[t]{3}{*}{$<0.001$} & Reference & \multirow[t]{3}{*}{$<0.001$} \\
\hline 1 & $1.12(1.05-1.19)$ & & $1.15(1.07-1.22)$ & \\
\hline$\geq 2$ & $1.33(1.17-1.51)$ & & $1.31(1.15-1.49)$ & \\
\hline \multicolumn{5}{|l|}{ Tumor location } \\
\hline Fundus & Reference & \multirow[t]{5}{*}{$<0.001$} & Reference & 0.03 \\
\hline Corpus & $1.07(0.95-1.20)$ & & $1.09(0.96-1.23)$ & \\
\hline Antrum/pylorus & $0.90(0.81-1.01)$ & & $0.99(0.88-1.11)$ & \\
\hline Curvatures & $0.95(0.85-1.07)$ & & $1.02(0.91-1.16)$ & \\
\hline Other & $1.07(0.97-1.18)$ & & $1.09(0.98-1.21)$ & \\
\hline Tumor grade & & & & \\
\hline Grade $1 / 2$ & Reference & $<0.001$ & Reference & $<0.001$ \\
\hline Grade $3 / 4$ & $1.23(1.14-1.31)$ & & $1.23(1.14-1.33)$ & \\
\hline Unknown & $1.34(1.23-1.46)$ & & $1.22(1.12-1.33)$ & \\
\hline Radiation therapy & & & & \\
\hline No & Reference & 0.60 & Reference & 0.23 \\
\hline Yes & $1.02(0.95-1.10)$ & & $1.05(0.97-1.13)$ & \\
\hline Metastasis & & & & \\
\hline Distant LNs only & Reference & $<0.001$ & Reference & $<0.001$ \\
\hline Peritoneal only & $1.20(1.09-1.33)$ & & $1.26(1.13-1.40)$ & \\
\hline Peritoneal \pm solid organ & $1.33(1.22-1.45)$ & & $1.23(1.13-1.34)$ & \\
\hline Distant LNs and peritoneal \pm solid organ & $1.48(1.34-1.64)$ & & $1.40(1.27-1.55)$ & \\
\hline Unknown & $1.12(0.99-1.27)$ & & $1.18(1.05-1.34)$ & \\
\hline Histology & & & & \\
\hline Adenocarcinoma NOS & Reference & $<0.001$ & Reference & 0.13 \\
\hline Intestinal & $0.80(0.71-0.90)$ & & $0.91(0.81-1.03)$ & \\
\hline Diffuse type, linitis plastica & $0.96(0.86-1.07)$ & & $0.99(0.88-1.11)$ & \\
\hline Signet-ring cell features & $1.07(1.01-1.13)$ & & $1.05(0.99-1.12)$ & \\
\hline
\end{tabular}


Table 3 continued

\begin{tabular}{|c|c|c|c|c|}
\hline & \multicolumn{2}{|c|}{ Unadjusted Cox regression $^{\mathrm{a}}$} & \multicolumn{2}{|c|}{ Multivariable adjusted Cox regression ${ }^{\mathrm{b}}$} \\
\hline & $\operatorname{HR}(95 \% \mathrm{CI})$ & $p$ value* & HR $(95 \% \mathrm{CI})$ & $p$ value* \\
\hline \multicolumn{5}{|l|}{ Chemotherapy type } \\
\hline Single agent & Reference & $<0.001$ & Reference & $<0.001$ \\
\hline Multiple agents & $0.78(0.71-0.85)$ & & $0.79(0.72-0.87)$ & \\
\hline Unknown & $0.74(0.65-0.84)$ & & $0.77(0.68-0.88)$ & \\
\hline
\end{tabular}

Hazard ratios (HR) with 95\% confidence intervals (CI; Wald type)

${ }^{a}$ Univariate Cox regression analysis

b Multivariable adjusted Cox regression analysis, full model

* $p$ from likelihood ratio test

$N O S$ not otherwise specified, $L N$ lymph node

\section{Subgroup analyses}

Subgroup analysis was performed excluding patients younger than 20 and older than 75 years. Assuming that patients who had a very poor prognosis were unlikely to undergo primary tumor resection, we did perform a subgroup analysis with exclusion of patients who survived less than 3 months (exclusion of 1919 patients). After full bipartite matching, the 2-year survival rate with and without primary tumor resection was $26.5 \%$ (95\% CI, $22.0-31.9 \%$ ) and $14.9 \%$ (95\% CI, 12.4-17.9\%), with a HR of 0.70 (95\% CI, 0.59-0.82; $p<0.001$ ). Similar results were also found after exact propensity score adjustment (data not shown). When further limiting this subgroup to patients with distant lymph node metastases only, 112 of 531 patients $(21.1 \%)$ underwent resection of the primary tumor, with a univariable HR for resection of 0.56 (95\% CI, 0.45-0.70; $p<0.001)$ and 0.52 (95\% CI, 0.41-0.66; $p<0.001$ ), respectively, after multivariable adjustment. Of 758 patients with peritoneal metastases, only 129 (17\%) underwent resection with an associated univariable HR for resection of $0.68(95 \% \mathrm{CI}, 0.55-0.83 ; p<0.001)$ and 0.66 (95\% CI, $0.53-0.83 ; p<0.001)$, respectively, after multivariable adjustment. The resection rate in patients with multiple metastases was lower, with 56 of 808 patients only $(6.9 \%)$.

\section{Discussion}

To the best of our knowledge, this is the first populationbased study investigating the association of OS and PTRaC versus chemotherapy alone among metastatic GC patients. The results indicate a significant survival benefit for patients who undergo PTRaC compared to patients who receive chemotherapy only. The significant survival benefit holds true, even after advanced statistical modeling including propensity score analysis and within-subgroup analysis limited to younger patients and excluding patients who survived less than 3 months, as well as after stratification by metastatic site.

For patients with metastatic GC, 5-year survival rates rarely exceed $5 \%$, and despite improvements in systemic therapies, median OS is less than 1 year [27-29]. Historically, perioperative mortality rates up to $20 \%$ made palliative gastrectomy unreasonable in the majority of metastatic GC cases, reserving this intervention for carefully selected symptomatic patients. However, perioperative mortality in the modern era is considerably reduced compared to earlier reports: 30-day mortality in our study was $1.3 \%$. Therefore, the rationale for resection of the primary tumor has renewed debate among experts in the field of GC [13, 15]. Furthermore, recent evidence suggests that for selected cancers, including colorectal and renal cell cancer, primary tumor resection in the metastatic settings can lead to meaningful improvements in OS [30, 31]. These benefits may become increasingly apparent as systemic chemotherapies and immunotherapies continue to improve.

The first strong evidence suggesting that primary tumor resection among GC patients may improve survival for patients with metastatic GC was reported in a retrospective subgroup analysis of the Dutch Gastric Cancer Trial [14]. In this analysis, patients who underwent gastrectomy in the metastatic setting had significantly improved survival (8.1 months) compared to patients undergoing palliative gastroenterostomy or laparotomy alone (5.4 months) $(p<0.001)$. In addition, two randomized controlled trials have prospectively evaluated the impact of primary tumor resection among stage IV GC patients. The recently published REGATTA trial was a multicenter Asian study comparing gastrectomy followed by chemotherapy (2-year OS, 25.1\%) versus chemotherapy alone (2-year OS, 31.7\%) among asymptomatic patients with metastatic gastric cancer [13]. This trial was ended prematurely after the first interim analysis, as the chance 
Table 4 Predictors of long-term survival ( $>2$ years) in patients who underwent primary tumor resection $(n=897)$

\begin{tabular}{|c|c|c|c|c|c|c|}
\hline & \multicolumn{4}{|c|}{ Univariable analysis } & \multicolumn{2}{|c|}{$\begin{array}{l}\text { Multivariable adjusted } \\
\text { logistic regression }\end{array}$} \\
\hline & $\begin{array}{l}\text { Total } \\
(n=897)\end{array}$ & $\begin{array}{l}\text { Long-term survivors } \\
(n=100)\end{array}$ & $\begin{array}{l}\text { Short-term survivors } \\
(n=797)\end{array}$ & $\overline{p \text { value* }}$ & OR $(95 \% \mathrm{CI})$ & $p$ value $^{\dagger}$ \\
\hline \multicolumn{7}{|l|}{ Gender } \\
\hline Male & $505(56.3)$ & $60(60.0)$ & $445(55.8)$ & \multirow[t]{2}{*}{0.429} & Reference & \multirow[t]{2}{*}{0.499} \\
\hline Female & $392(43.7)$ & $40(40.0)$ & $352(44.2)$ & & $0.85(0.53-1.36)$ & \\
\hline \multicolumn{7}{|l|}{ Age } \\
\hline$<50$ years & $183(20.4)$ & $12(12.0)$ & $171(21.5)$ & \multirow[t]{4}{*}{0.156} & Reference & \multirow[t]{4}{*}{0.030} \\
\hline 50-64 years & $380(42.4)$ & $49(49.0)$ & $331(41.5)$ & & $2.61(1.30-5.60)$ & \\
\hline $65-79$ years & $287(32.0)$ & $33(33.0)$ & 254 (31.9) & & $2.36(1.10-5.36)$ & \\
\hline $80+$ years & $47(5.2)$ & $6(6.0)$ & $41(5.1)$ & & 3.95 (1.16-12.6) & \\
\hline \multicolumn{7}{|l|}{ Race } \\
\hline White & $568(63.3)$ & $48(48.0)$ & $520(65.2)$ & \multirow[t]{3}{*}{0.003} & Reference & \multirow[t]{3}{*}{0.030} \\
\hline Black & $201(22.4)$ & $31(31.0)$ & $170(21.3)$ & & $1.71(0.99-2.92)$ & \\
\hline Other/unknown & $128(14.3)$ & $21(21.0)$ & $107(13.4)$ & & $2.08(1.11-3.80)$ & \\
\hline \multicolumn{7}{|l|}{ Year of diagnosis } \\
\hline 2006-2008 & $430(47.9)$ & $34(34.0)$ & $396(49.7)$ & \multirow[t]{3}{*}{0.012} & Reference & \multirow[t]{3}{*}{0.247} \\
\hline 2009-2010 & $226(25.2)$ & $31(31.0)$ & $195(24.5)$ & & $1.64(0.88-2.99)$ & \\
\hline 2011-2012 & $241(26.9)$ & $35(35.0)$ & $206(25.8)$ & & $1.57(0.81-3.01)$ & \\
\hline \multicolumn{7}{|l|}{ Charlson-Deyo Score } \\
\hline 0 & $676(75.4)$ & $79(79.0)$ & $597(74.9)$ & \multirow[t]{3}{*}{0.546} & Reference & \multirow[t]{3}{*}{0.276} \\
\hline 1 & $171(19.1)$ & $15(15.0)$ & $156(19.6)$ & & $0.65(0.33-1.19)$ & \\
\hline$\geq 2$ & $50(5.6)$ & $6(6.0)$ & $44(5.5)$ & & $1.35(0.47-3.37)$ & \\
\hline \multicolumn{7}{|l|}{ Tumor location } \\
\hline Fundus & $34(3.8)$ & $1(1.0)$ & $33(4.1)$ & \multirow[t]{5}{*}{0.020} & Reference & \multirow[t]{5}{*}{0.046} \\
\hline Corpus & $94(10.5)$ & $4(4.0)$ & $90(11.3)$ & & $0.23(0.01-1.19)$ & \\
\hline Antrum/pylorus & $283(31.5)$ & $38(38.0)$ & $245(30.7)$ & & $0.34(0.09-0.92)$ & \\
\hline Curvatures & $147(16.4)$ & $23(23.0)$ & $124(15.6)$ & & $1.31(0.71-2.40)$ & \\
\hline Other & $339(37.8)$ & $34(34.0)$ & $305(38.3)$ & & $0.97(0.56-1.67)$ & \\
\hline \multicolumn{7}{|l|}{ Tumor grade } \\
\hline Grade $1 / 2$ & $152(16.9)$ & $24(24.0)$ & $128(16.1)$ & \multirow[t]{3}{*}{0.110} & Reference & \multirow[t]{3}{*}{0.804} \\
\hline Grade $3 / 4$ & 705 (78.6) & $71(71.0)$ & $634(79.5)$ & & $0.87(0.49-1.60)$ & \\
\hline Unknown & $40(4.5)$ & $5(5.0)$ & $35(4.4)$ & & $1.18(0.33-3.63)$ & \\
\hline Resection margin & & & & & & \\
\hline R0 & $479(53.4)$ & $73(73.0)$ & $406(50.9)$ & $<0.001$ & Reference & 0.002 \\
\hline $\mathrm{R}+$ & $381(42.5)$ & $24(24.0)$ & $357(44.8)$ & & $0.42(0.24-0.70)$ & \\
\hline $\mathrm{Rx}$ & $37(4.1)$ & $3(3.0)$ & $34(4.3)$ & & $0.37(0.08-1.17)$ & \\
\hline Radiation therapy & & & & & & \\
\hline No & $739(82.4)$ & $71(71.0)$ & $668(83.8)$ & 0.002 & Reference & $<0.001$ \\
\hline Yes & $158(17.6)$ & $29(29.0)$ & $129(16.2)$ & & $2.74(1.57-4.73)$ & \\
\hline Metastasis & & & & & & \\
\hline Distant LNs only & 125 (13.9) & $21(21.0)$ & $104(13.0)$ & 0.005 & Reference & 0.05 \\
\hline Peritoneal only & $142(15.8)$ & $16(16.0)$ & $126(15.8)$ & & $0.62(0.27-1.42)$ & \\
\hline Peritoneal \pm solid organ & $470(52.4)$ & $39(39.0)$ & $431(54.1)$ & & $0.46(0.25-0.88)$ & \\
\hline Distant LNs and peritoneal \pm solid organ & $68(7.6)$ & $6(6.0)$ & $62(7.8)$ & & $0.36(0.11-0.98)$ & \\
\hline Unknown & $92(10.3)$ & $18(18.0)$ & $74(9.3)$ & & $0.95(0.41-2.19)$ & \\
\hline
\end{tabular}


Table 4 continued

\begin{tabular}{|c|c|c|c|c|c|c|}
\hline & \multicolumn{4}{|c|}{ Univariable analysis } & \multicolumn{2}{|c|}{$\begin{array}{l}\text { Multivariable adjusted } \\
\text { logistic regression }\end{array}$} \\
\hline & $\begin{array}{l}\text { Total } \\
(n=897)\end{array}$ & $\begin{array}{l}\text { Long-term survivors } \\
(n=100)\end{array}$ & $\begin{array}{l}\text { Short-term survivors } \\
(n=797)\end{array}$ & $p$ value* & OR $(95 \% \mathrm{CI})$ & $p$ value $^{\dagger}$ \\
\hline \multicolumn{7}{|l|}{ Histology } \\
\hline Adenocarcinoma NOS & $463(51.6)$ & $66(66.0)$ & $397(49.8)$ & 0.002 & Reference & 0.041 \\
\hline Intestinal & $88(9.8)$ & $13(13.0)$ & $75(9.4)$ & & $0.94(0.43-1.94)$ & \\
\hline Diffuse type, linitis plastica & $75(8.4)$ & $6(6.0)$ & $69(8.7)$ & & $0.60(0.21-1.46)$ & \\
\hline Signet-ring cell features & $271(30.2)$ & $15(15.0)$ & $256(32.1)$ & & $0.41(0.21-0.76)$ & \\
\hline \multicolumn{7}{|l|}{ Chemotherapy sequence } \\
\hline Chemotherapy first & $158(17.6)$ & $16(16.0)$ & $142(17.8)$ & 0.039 & Reference & 0.042 \\
\hline Surgery first & $677(75.5)$ & $71(71.0)$ & $606(76.0)$ & & $0.99(0.54-1.92)$ & \\
\hline Chemotherapy before and after & $62(6.9)$ & $13(13.0)$ & $49(6.1)$ & & $2.76(1.11-6.85)$ & \\
\hline \multicolumn{7}{|l|}{ Chemotherapy type } \\
\hline Single agent & $122(13.6)$ & $11(11.0)$ & $111(13.9)$ & 0.527 & Reference & 0.205 \\
\hline Multiple agents & $686(76.5)$ & $81(81.0)$ & $605(75.9)$ & & $1.88(0.91-4.21)$ & \\
\hline Agent unknown & $89(9.9)$ & $8(8.0)$ & $81(10.2)$ & & $1.35(0.46-3.84)$ & \\
\hline
\end{tabular}

to find an OS benefit at the end of the study for the gastrectomy plus chemotherapy group compared to chemotherapy only was only $13.2 \%$. The inclusion criteria to the REGATTA trial were very strict and limited to asymptomatic patients with metastatic sites specifically limited to peritoneum in the diaphragm or peritoneum caudal to the transverse colon without massive ascites or intestinal obstruction or with paraaortic lymph node metastases above the celiac axis or below the inferior mesenteric artery. Although it was impossible to apply such strict inclusion criteria to this retrospective population-based analysis using NCDB, an OS benefit for patients with 'distant lymph node metastases only' and 'peritoneal metastases only' persisted, which, with all its limitations, might diverge from this recently published trial from the East. In contrast, the American single-center GYMSSA trial was intended to compare the combination of primary tumor resection, metastasectomy, hyperthermic intraperitoneal chemotherapy (HIPEC), and systemic chemotherapy with systemic chemotherapy alone [32]. Although this study was unfortunately terminated because of poor accrual, preliminary results showed that median OS among patients with systemic therapy was 4.3 months, compared to 11.3 months after multidisciplinary treatment including gastrectomy. Despite the inconclusive nature of the GYMSSA study, its limited data suggest resection in addition to systemic therapy may benefit certain carefully selected patients within the Western population. The importance of this open research question is even further highlighted by a study assessing "cytoreduction, gastrectomy, and hyperthermic intraperitoneal chemoperfusion (HIPEC) in gastric adenocarcinoma and carcinomatosis or positive cytology," which recently began to recruit patients in the US (NCT02891447).

Differences between Western and Eastern GC are well documented. Patients in Western countries often present with more advanced stage disease as compared to Eastern countries where screening programs exist [33, 34]; moreover, for reasons that remain poorly understood, Asian patients may in fact have a better stage-for-stage prognosis than Western patients [20, 21, 35]. Recent data also suggest an ethnic difference in responses to chemotherapy, again implying a biological difference in disease process [17-19]. Together, these findings question whether the negative results of the REGATTA trial are generalizable to Western populations.

Further supporting our analysis are several smaller retrospective analyses and meta-analyses that have reported improved long-term outcomes for patients who underwent PTRaC compared to chemotherapy alone [11, 15, 28, 36]. Multiple independent predictors for OS are discussed within these studies, which are also corroborated through our analysis. Together, they lend greater support to the argument for a potential role of PTRaC and, because of our 
use of a large nationwide database and advanced statistical modeling, we potentially negate some of the inherent publication bias found in single-center retrospective analyses $[11,28,36]$.

In evaluating the palliative nature of interventions for patients with metastatic GC, the importance of qualityof-life measures (QoL) cannot be overemphasized. To date, however, no prospective data exist focusing on QoL measures among patients undergoing primary tumor resection for metastatic GC, and no such data are currently available from the NCDB. However, within a single-center cohort study, QoL measures including time to return to normal daily activities, time to return to oral nutritional intake, and frequency of vomiting and gastrointestinal bleeding were assessed between patients undergoing gastrectomy versus non-resectional procedures. In this study, all three of these QoL endpoints were improved for patients who underwent palliative resection [37]. This finding suggests that in addition to an OS improvement for primary tumor resection, there may also be benefits in patient-reported outcomes, necessitating that any future prospective studies prioritize QoL measures as study endpoints.

\section{Limitations}

The large number of patients with metastatic GC in this population-based analysis allowed for advanced statistical modeling, in which adjustments were made for differences in baseline characteristics between study groups. However, given the inherent limitations of registry data on variable granularity, hidden selection bias remains a potential limitation. For example, detailed information about tumor burden and metastatic site among patients with metastatic disease is missing; therefore, surrogates for tumor burden as described within the Methods section were evaluated. Despite this attempt to adjust for unavailable data, it is likely that patients with limited metastatic disease are overrepresented within the group of patients undergoing PTRaC. Furthermore, we were not able to stratify patients as to whether the reason for surgical resection was based on primary tumor-related symptoms. Given that $75.5 \%$ of the patients received chemotherapy only after surgery might imply that those patients suffered from symptomatic gastric lesions. However, this correlation remains hypothetical, but should be addressed in any further investigation if possible. In analogy to most other studies, we also lack information about QoL measures. In addition, information on HER2-status is not captured in the NCDB, and therefore stratified analyses between these tumor factors were not possible.

\section{Conclusion}

Significant uncertainty remains about the potential benefits of primary tumor resection in Western patients with metastatic GC. Our results support the need for further prospective trials investigating the influence of PTRaC on survival among patients from the US and Europe. Our data strongly suggest that there exists, at the very least, a subset of carefully selected patients for which PTRaC can improve OS. Until data from Western prospective randomized studies become available, patients with metastatic GC should continue to be evaluated within a multidisciplinary team that includes surgeons, and primary tumor resection should be considered cautiously on an individual basis.

\section{Compliance with ethical standards}

Conflict of interest The authors declare that they have no conflict of interest.

Funding This research did not receive any specific grant from funding agencies in the public, commercial, or not-for-profit sectors.

Informed consent For this type of study, formal consent is not required.

Human and animal rights statement All procedures followed were in accordance with the ethical standards of the responsible committee on human experimentation (institutional and national) and with the Helsinki Declaration of 1964 and later versions.

\section{References}

1. Zhu AL, Sonnenberg A. Is gastric cancer again rising? J Clin Gastroenterol. 2012;46(9):804-6.

2. Best LM, Mughal M, Gurusamy KS. Laparoscopic versus open gastrectomy for gastric cancer. Cochrane Database Syst Rev. 2016;3:CD011389.

3. http://globocan.iarc.fr/Pages/fact_sheets_population.aspx. Last accessed 28 Nov 2016.

4. Siegel RL, Miller KD, Jemal A. Cancer statistics, 2016. CA Cancer J Clin. 2016;66(1):7-30.

5. Inoue M, Tsugane S. Epidemiology of gastric cancer in Japan. Postgrad Med J. 2005;81(957):419-24.

6. Wanebo HJ, et al. Cancer of the stomach. A patient care study by the American College of Surgeons. Ann Surg. 1993;218(5):583-92.

7. Izuishi K, Mori H. Recent strategies for treating stage IV gastric cancer: roles of palliative gastrectomy, chemotherapy, and radiotherapy. J Gastrointest Liver Dis. 2016;25(1):87-94.

8. Cunningham D, et al. Perioperative chemotherapy versus surgery alone for resectable gastroesophageal cancer. $\mathrm{N}$ Engl $\mathrm{J}$ Med. 2006;355(1):11-20.

9. Macdonald JS, et al. Chemoradiotherapy after surgery compared with surgery alone for adenocarcinoma of the stomach or gastroesophageal junction. N Engl J Med. 2001;345(10):725-30.

10. Japanese Gastric Cancer Association. Japanese gastric cancer treatment guidelines 2010 (ver. 3). Gastric Cancer. 2011;14(2):113-23. 
11. He MM, et al. The role of non-curative surgery in incurable, asymptomatic advanced gastric cancer. PLoS One. 2013;8(12):e83921.

12. Hallissey MT, et al. Palliative surgery for gastric cancer. Cancer (Phila). 1988;62(2):440-4.

13. Fujitani K, et al. Gastrectomy plus chemotherapy versus chemotherapy alone for advanced gastric cancer with a single non-curable factor (REGATTA): a phase 3, randomised controlled trial. Lancet Oncol. 2016;17(3):309-18.

14. Hartgrink $\mathrm{HH}$, et al. Value of palliative resection in gastric cancer. Br J Surg. 2002;89(11):1438-43.

15. Ebinger SM, et al. Modest overall survival improvements from 1998 to 2009 in metastatic gastric cancer patients: a populationbased SEER analysis. Gastric Cancer. 2016;19(3):723-34.

16. Bollschweiler E, et al. Treatment of early gastric cancer in the Western World. World J Gastroenterol. 2014;20(19):5672-8.

17. Ohtsu A, Yoshida S, Saijo N. Disparities in gastric cancer chemotherapy between the East and West. J Clin Oncol. 2006;24(14):2188-96.

18. Kim J, et al. Disparities in gastric cancer outcomes among Asian ethnicities in the USA. Ann Surg Oncol. 2009;16(9):2433-41.

19. Kim J, et al. Race and ethnicity correlate with survival in patients with gastric adenocarcinoma. Ann Oncol. 2010;21(1):152-60.

20. Kirchoff DD, et al. Overall survival is impacted by birthplace and not extent of surgery in Asian Americans with resectable gastric cancer. J Gastrointest Surg. 2015;19(11):1966-73.

21. Byfield SA, et al. Treatment and outcomes of gastric cancer among United States-born and foreign-born Asians and Pacific Islanders. Cancer (Phila). 2009;115(19):4595-605.

22. American College of Surgeons. About the National Cancer Database. https://www.facs.org/quality-programs/cancer/ncdb/ about. Last visited: November 2016].

23. Ebinger SM, et al. Modest overall survival improvements from 1998 to 2009 in metastatic gastric cancer patients: a populationbased SEER analysis. Gastric Cancer. 2015;19(3):723-34.

24. Grambsch P. Proportional hazards tests and diagnostics based on weighted residuals. Biometrika. 1994;81:515-6.
25. Ho D. MatchIt: nonparametric preprocessing for parametric causal inference. J Stat Softw. 2011;42(1):1-28.

26. Hansen B. Optimal full matching and related designs via network flows. J Comput Graph Stat. 2006;15(3):609-27.

27. Chang YR, et al. The value of palliative gastrectomy in gastric cancer with distant metastasis. Ann Surg Oncol. 2012;19(4):1231-9.

28. Lasithiotakis K, et al. Gastrectomy for stage IV gastric cancer. a systematic review and meta-analysis. Anticancer Res. 2014;34(5):2079-85.

29. Sun W, Yan L. Gastric cancer: current and evolving treatment landscape. Chin J Cancer. 2016;35(1):83.

30. Tarantino I, et al. Prognostic relevance of palliative primary tumor removal in 37,793 metastatic colorectal cancer patients: a population-based, propensity score-adjusted trend analysis. Ann Surg. 2015;262(1):112-20.

31. Flanigan RC, et al. Nephrectomy followed by interferon alfa- $2 b$ compared with interferon alfa- $2 \mathrm{~b}$ alone for metastatic renal-cell cancer. N Engl J Med. 2001;345(23):1655-9.

32. Rudloff U, et al. Impact of maximal cytoreductive surgery plus regional heated intraperitoneal chemotherapy (HIPEC) on outcome of patients with peritoneal carcinomatosis of gastric origin: results of the GYMSSA trial. J Surg Oncol. 2014;110(3):275-84.

33. Shin A, Kim J, Park S. Gastric cancer epidemiology in Korea. J Gastric Cancer. 2011;11(3):135-40.

34. Schlansky B, Sonnenberg A. Epidemiology of noncardia gastric adenocarcinoma in the United States. Am J Gastroenterol. 2011;106(11):1978-85.

35. Wang A, et al. Stage-specific prognostic effect of race in patients with resectable gastric adenocarcinoma: an 8-institution study of the US Gastric Cancer Collaborative. J Am Coll Surg. 2016;222(4):633-43.

36. Sun J, et al. Clinical significance of palliative gastrectomy on the survival of patients with incurable advanced gastric cancer: a systematic review and meta-analysis. BMC Cancer. 2013;13:577.

37. Samarasam I, et al. Palliative gastrectomy in advanced gastric cancer: is it worthwhile? ANZ J Surg. 2006;76(1-2):60-3. 